\title{
Experimental Evaluation of All-Optical Asynchronous and Synchronous Memories
}

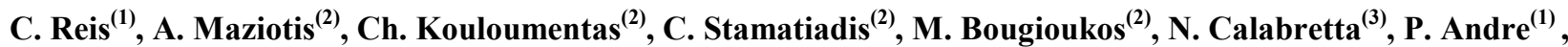 \\ R. Dionisio ${ }^{(1)}$, B.Neto ${ }^{(1)}$, Harm. J. S. Dorren ${ }^{(3)}$, H. Avramopoulos ${ }^{(2)}$ and A. Teixeira ${ }^{(1)}$ \\ (1) Instituto de Telecomunicações, Universidade de Aveiro, Portugal creis@av.it.pt \\ (2) National Technical University of Athens - School of Electrical and Computer Engineering, \\ 9 Iroon Polytechniou Street, Zografou 15773 - Athens, Greece \\ (3) Eindhoven University of Technology, Netherlands
}

\begin{abstract}
We experimentally demonstrate the accuracy of an all-optical $S$-R latch and an optical $S$ - $R$ flip-flop based on hybrid integrated Mach-Zehnder Interferometers, with Semiconductor Optical Amplifier in each arm (MZI-SOA). The performance of both bistable devices will be studied and compared in terms of extinction ratio and switching times.
\end{abstract}

Keywords: Optical switching, optical flip-flop, optical signal processing, semiconductor optical amplifier, Mach-Zenhder interferometer (MZI), memory unit.

\section{Introduction}

Optical packet switching (OPS) can offer increased network capacity, with low power consumption [1].

In comparison to electronic switching, OPS offers advantages in terms of transparency, foot-print and can reduce latency for telecommunication networks [2].

Besides all the known applications that an optical bistable device can fulfil such as clock dividers, optical counters and shift registers, optical buffers are essential building blocks for implementing an all-optical packet switch.

An optical bistable have at least two stable states and the information present at the inputs is transferred to the output, according to its truth table.

An optical bistable is a sequential device therefore its outputs not only depend of the input values in a considered instant, but also depend of information from a previous state.

Different technologies have been proposed to provide optical buffering such as the use of, a SOA based MachZenhder interferometer with a feedback loop [3], two coupled identical laser diodes [4], fiber-delay lines [5], two coupled fiber ring lasers [6] or two coupled MZISOA [7].

Among these approaches, the ones that use active interferometric devices attracts increasing attention because they require low energy, have high compactness and extinction ratio (ER), as well the potential for further optical integration.

In [8], an optical RAM memory exploiting the regeneration and integration proprieties of hybrid MZISOAs was implemented with true random access $\mathrm{read} /$ write capability.

In this paper, a performance comparison between two optical bistable configurations, based on the same hybrid platform, will be conducted.

The paper is organized as follows. In Section II, the optical S-R latch and the optical S-R flip-flop are introduced and qualitatively assessed. In Section III, quantitative achievements are presented and discussed.

\section{Optical S-R latch and optical S-R flip-flop}

Most of the optical bistable devices known in the literature are asynchronous S-R latches, and any change of information in the inputs is transmitted, immediately, to the output, according to its truth table (Table 1), which not allows them to work as a finite state machine.

\begin{tabular}{|c|c|c|}
\hline $\mathrm{R}$ & $\mathrm{S}$ & $\mathrm{Q}$ \\
\hline \hline 0 & 0 & Last $\mathrm{Q}$ \\
\hline 0 & 1 & 1 \\
\hline 1 & 0 & 0 \\
\hline 1 & 1 & Forbidden \\
\hline
\end{tabular}

Tab. 1: Truth table of S-R latch

Fig. 1 illustrates the experimental setup of the optical S-R latch. The integrated device comprises two coupled MZI-SOA powered by two continuous wave $(\mathrm{CW})$ input signals at different wavelengths. The logic level obtained in the output is determined by the dominant wavelength. By injecting set and reset optical pulses, the dynamic operation occurs and the latch S-R toggles its logical state [7]. 


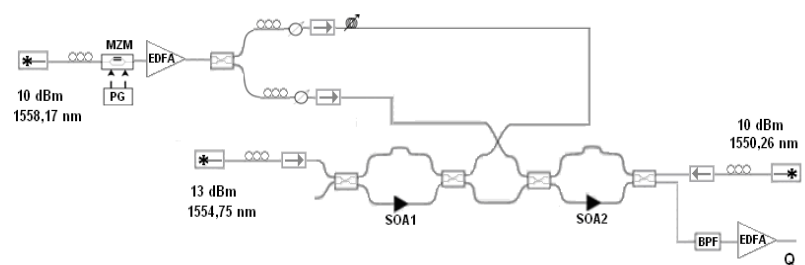

Fig. 1: Optical asynchronous S-R latch: MZM: MachZehnder modulator; EDFA: Erbium Doped Fiber Amplifier; BPF: band pass filter; SOA: semiconductor optical amplifier; PG: pattern generator.

In order to generate the set and reset optical pulses, an external cavity laser peaking at $1558,17 \mathrm{~nm}$ were used, followed by a polarization controller and a Mach-Zehnder external modulator. The NRZ data signal, generated by an Anritsu MP 1763C, is then amplified by an Erbium Doped Fiber Amplifier and split into two equal parts using a $3 \mathrm{~dB}$ coupler.

Different set and reset patterns are obtained by delaying the signals by $11,6 \mathrm{~ns}$.

The dynamic operation of the S-R latch was experimentally demonstrated by toggling the state of the latch through the injection of set and reset optical pulses, as shown in Fig. 2.

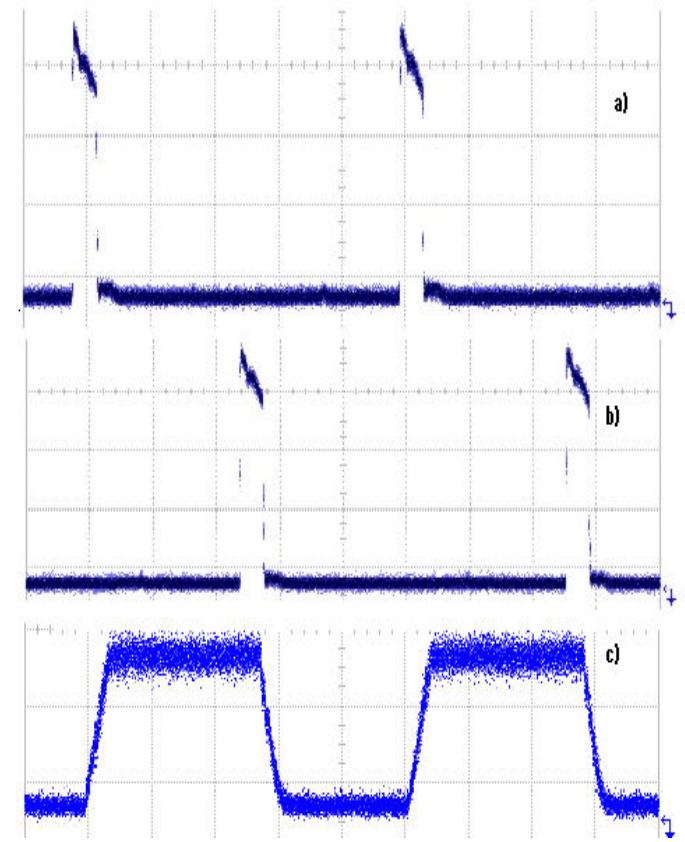

Fig. 2: Experimental results for asynchronous S-R latch: a) Set signal; b) Reset signal; c) Latch output. The vertical scale is arbitrary and the horizontal scale is $5 \mathrm{~ns} /$ div.

The observed results in the oscilloscope are in agreement with the truth table for an asynchronous S-R latch.

In some applications, it is very important that exists a synchronization signal responsible for controlling the enabling of the latch, making the latch sensitive to the values present in the set and reset inputs.

A synchronous all-optical S-R flip-flop, shown in Fig.3, was implemented in [9] using two coupled fiber ring lasers and additional logic gates exploiting the nonlinear effects of semiconductor optical amplifiers (SOA).

The logic AND operation was realized using integrated SOA-based Mach-Zehnder interferometers, after balanced them.

The powers of the continuous wave signals (CW) used in MZI-SOA1 and MZI-SOA2 were set to adjust the gain of the SOAs and the outputs of AND1 and AND2 were obtained at the switching ports of the MZI-SOAs.

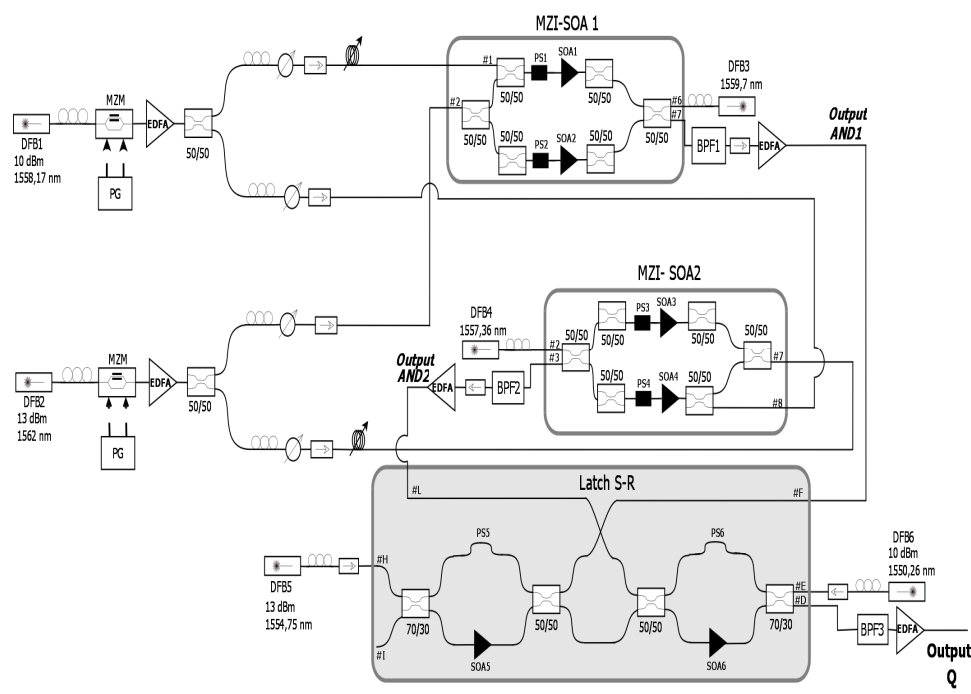

Fig. 3: Optical synchronous S-R Flip-Flop: MZM: MachZehnder modulator; EDFA: Erbium Doped Fiber Amplifier; BPF: band pass filter; SOA: semiconductor optical amplifier; PG: pattern generator.

The truth table of the clocked S-R flip-flop is the same as the asynchronous S-R latch but the information present in the inputs of the bistable only produce effects on its output throughout all the clock pulse period. If CLK $=0$, the flip-flop maintains its previous state (last Q).

The clock signal was generated by an external cavity laser, peaking at $1562 \mathrm{~nm}$ and $13 \mathrm{dBm}$ of optical power, followed by a Mach-Zehnder optical modulator (MZM).

The set and reset input signals were the same as the ones used to test the asynchronous bistable.

In Fig.4, we experimentally demonstrate the operation of a synchronous all-optical Set-Reset (S-R) flip-flop. Fig. 4 illustrates that only when the clock signal is in the high level the synchronous S-R flip-flop changes its state, otherwise the flip-flop maintains its previous value. 

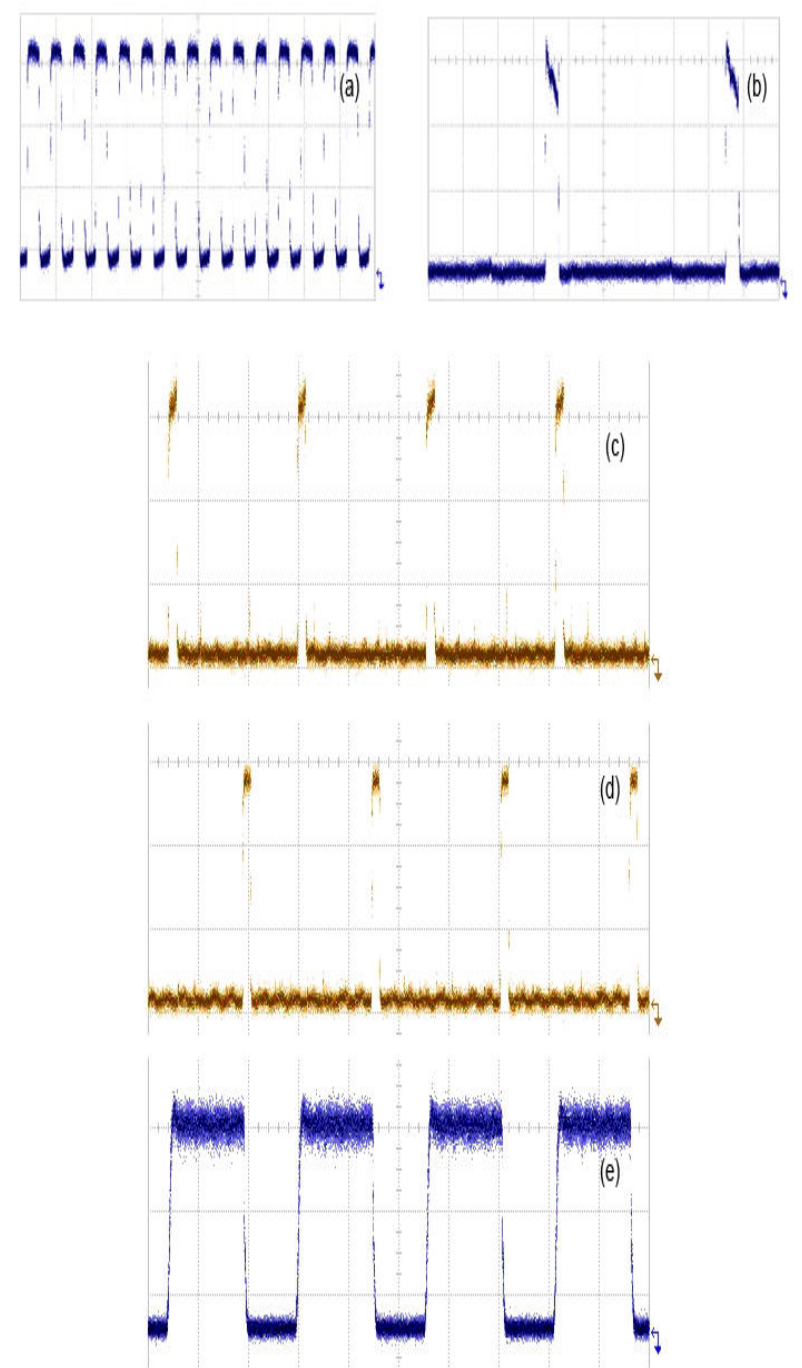

Fig. 4 Experimental results for synchronous S-R flip-flop: a)

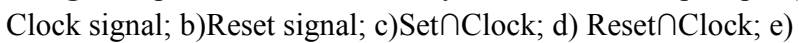

Flip-Flop output. The vertical scale is arbitrary and the horizontal scale is $5 \mathrm{~ns} / \mathrm{div}$ for a) b) figures and $10 \mathrm{~ns} / \mathrm{div}$ for c)d)e).

\section{Quantitative results for bistable devices}

The following table summarizes the experimental results obtained, to perform a comparison between the two different bistable devices.

Analysing Table 2, we observe that injecting identical set and reset signals, with synchronous bistable devices we achieved better extinction ratios and is possible to reduced rise and fall times (hundreds of $\mathrm{ps}$ ).

\begin{tabular}{|c|c|c|}
\hline Figure of Merit & $\begin{array}{c}\text { All-optical S-R } \\
\text { Latch }\end{array}$ & $\begin{array}{c}\text { All-optical S- } \\
\text { R Flip-Flop }\end{array}$ \\
\hline \hline Rise Time & $1,029 \mathrm{~ns}$ & $430 \mathrm{ps}$ \\
\hline Fall Time & $857 \mathrm{ps}$ & $420 \mathrm{ps}$ \\
\hline $\begin{array}{c}\text { Exctintion Ratio } \\
\text { (ER) }\end{array}$ & $12,7 \mathrm{~dB}$ & $18 \mathrm{~dB}$ \\
\hline
\end{tabular}

Tab.2: Comparison between asynchronous and synchronous one-bit memories.

\section{Conclusions}

An all-optical flip-flop is a key element for routing optical packets or burst.

In this paper, we experimentally compared the switching dynamics and system performance of two bistable devices - one asynchronous and the other synchronous.

By analyzing the experimental results obtained, we can conclude that synchronous bistables would be a better choice because allow high output extinction ratios and faster switching times.

Acknowledgments: This work was supported by the Network of Excellence EURO-FOS funded by the European Commission and by the Portuguese Scientific Project PANORAMA (ADI 2009/003144).

\section{References}

[1] N. Calabretta et al., "All-optical techniques enabling packet switching with label processing and label rewriting", Journal of Telecommunications and Information Technology, pp. 20-2, 2009(1). Invited paper.

[2] D. J. Blumenthal et al., "Optical Packet Switching and Associated Optical Signal Processing", IEEE LEOS Summer TuG2-17, November 2002.

[3\} R. Clavero et al., "All-optical flip-flop based on an active Mach-Zehnder interferometer with a feedback loop", Optics Letters, vol. 30, no. 21, pp. 2861-2863, November 2005.

[4] M.T. Hill et al., "All-optical flip-flop based on coupled lasers diodes”, IEEE J. Quantum Electron., vol. 37, n³, pp. 405-413, March 2001.

[5] L. Li et al., "A Novel Fiber Delay Line Buffering Architecture for Optical Packet Switching", IEEE GLOBECOM, pp. 2809-2813, December 2003.

[6] J. Wang et al., "All-optical clocked flip-flops exploiting SOA-based ST latches and logic gates", OSC 2009, LNCS 5882, pp. 5-18, 2009.

[7] Y. Liu et al.,"Packaged and hybrid integrated all-optical flip-flop memory", Electron. Lett., vol. 42, No. 24, pp. 1399-1400, November 2006. 
[8] N. Pleros et al.,"Optical Static RAM CelI", IEEE Photonics Technology Letters, Vol. 21, No. 2, January 2009.

[9] C. Reis, "All-optical synchronous S-R flip-flop based on active interferometric devices" Electron. Lett., Vol. 46 No. 10, May 2010. 